\title{
A new ionospheric tomographic algorithm - constrained multiplicative algebraic reconstruction technique (CMART)
}

\author{
Wen DeBAO ${ }^{1,2, *}$ and LiU SANZHi ${ }^{3}$ \\ ${ }^{1}$ School of Traffic and Transportation Engineering, Changsha University of Science \& Technology, \\ Changsha 410 004, China. \\ ${ }^{2}$ Key Laboratory of Dynamic Geodesy, Institute of Geodesy and Geophysics, Chinese Academy of Sciences, \\ Wuhan 430 07r, China. \\ ${ }^{3}$ Department of Surveying, Tongji University Shanghai, Shanghai 200 092, China. \\ *e-mail: wdbwhigg@163.com
}

For the limitation of the conventional multiplicative algebraic reconstruction technique (MART), a constrained MART (CMART) is proposed in this paper. In the new tomographic algorithm, a popular two-dimensional multi-point finite difference approximation of the second order Laplacian operator is used to smooth the electron density field. The feasibility and superiority of the new method are demonstrated by using the numerical simulation experiment. Finally, the CMART is used to reconstruct the regional electron density field by using the actual GNSS data under geomagnetic quiet and disturbed days. The available ionosonde data from Beijing station further validates the superiority of the new method.

\section{Introduction}

Methods of satellite radio tomography of the ionosphere have been under intense development in recent years. The first suggestions that satellite navigation system could be used for such a purpose were made more than 20 years ago (Austen et al 1988). Then many experimental tomographic campaigns were conducted (Andreeva et al 1990; Kunitsyn and Tereshchenko 1992; Kunitake et al 1995; Markkanen et al 1995; Mitchell et al 1995; Pryse et al 1995; Huang et al 1998). In these studies, the used observation data are radio signal from Navy Navigation Satellite System (NNSS). Although these studies are useful for understanding the ionospheric structure, only twodimensional ionospheric structure within a cross section of latitude and height can be obtained. However, three-dimensional ionospheric electron density (IED) distributions are very crucial to obtain high-precision ionospheric delay correction. The present development of Global Navigation Satellite Systems (GNSS) receivers, both on ground and onboard satellites, and the advent of new GNSS satellite constellations is opening a new era for ionosphere imaging by increasing greatly the amount of data available for retrieval of time varying three-dimensional electron density distributions (Wen 2007, 2010; Wen et al 2007a; Garcia and Crespon 2008).

Unlike traditional computer tomographic problems widely used in medical applications, where transform-based reconstruction techniques are popular (Kak and Slaney 1988), many GNSSbased ionosphere tomographic methods are based on ray approximation. The main reason is that we usually do not have the freedom to choose measurement ray paths crossing the region of interest in GNSS-based ionospheric tomography. GNSSbased ionospheric tomography typically solves the

Keywords. GNSS; ionosphere; electron density; tomography; CMART; MART; geophysics; remote sensing; atmospheric sciences. 
ill-conditioned inverse problem by a direct or iterative inversion of the ray projection matrices. In addition to the incomplete ray geometry, background noise and measurement noise are inevitably attached to the observed phase difference and Faraday rotation. Therefore, radio tomography is more ill-posed compared to the widely known computer tomography in medical applications (Zhai and Cummer 2005).

To resolve the ill-posed problem in GNSS-based ionospheric tomography, a number of tomographic algorithms have been developed in the past. One of the conventional algorithms is MART because of its rapid convergence. However, MART incorporates prior information into each pixel to cope with the ill-posed problem. For those pixels without any ray paths crossing them in the probed region, the final results are the same as the initial values, which are obtained from empirical ionospheric models that reflect month-average variation of the ionosphere, so the reconstruction accuracy can be severely limited. For the above problem, Pryse et al (1998) presented a scheme based on singular value decomposition (SVD) followed by MART, which is defined as a combined algorithm (CA) in this work. Although this method improves the accuracy of the reconstructed IED, the computational efficiency is low because of the sparsity and the large dimension of the coefficient matrix. In order to resolve the limitations of the conventional tomographic algorithms, a CMART is presented in this paper. In this new method, the constraint is imposed according to the two-dimensional multi-point finite difference approximation of the second order Laplacian operator in the horizontal direction. Numerical simulation experiment has demonstrated the feasibility of the new method and its superiority to the above algorithms. Finally, the new method is applied to reconstruct the IED distributions using the regional GNSS observation (i.e., GPS) over China.

\section{Tomographic formulation}

As is well known, one measurable parameter of the ionosphere is the total electron content (TEC), which is the line integral of IED along ray propagation path. It can be written as (Wen et al 2007b, 2008):

$$
\mathrm{TEC}=\int_{l} N(s) d s
$$

where $N(s)$ is the electron density along the propagation path $l$ between a satellite and a receiver.

In general, TEC along some path can be approximated as a finite sum of shorter integrals along segments of $l_{i}$. To simplify the computation, the reconstructed region is first discretized into some small pixels. Assuming the distribution of electron density to be uniform in discrete regions, equation (1) can be formulated as:

$$
y_{i}=\sum_{j=1}^{n} A_{i j} x_{j}+e_{i}, \quad i=1,2, \ldots, m .
$$

Equation (2) can be generally written in a simple matrix notation as:

$$
y_{m \times 1}=A_{m \times n} x_{n \times 1}+e_{m \times 1},
$$

where $n$ is the number of voxels in the image, $m$ is the number of TEC measurements, $y$ is a column vector of the $m$ known TEC measurements, $A$ is a matrix containing all the lengths of the $m$ ray paths traversing the corresponding $n$ voxels, $x$ is the vector consisting of all the unknown electron densities in all the voxels, and $e$ is a column vector associated with the discretization errors and measurement noises.

\section{Constrained MART}

To reconstruct the IED distribution, a reasonable algorithm should be selected. MART is very attractive due to its advantage over algebraic reconstruction technique in determining the electron densities to avoid unreasonable negative values and rapid convergence. In general, the MART algorithm is iterated cyclically and can be implemented as follows:

$$
x_{j}^{k+1}=x_{j}^{k}\left(\frac{y_{i}}{a_{i} \cdot x^{k}}\right)^{\lambda_{k} A_{i j}}
$$

where $x_{j}^{k+1}$ is the $j$ th member of the vector of pixels $x^{k+1}, k$ is the number of changes to $x, a_{i}$ is the $i$ th row of matrix $A, \lambda_{k}$ is the relaxation parameter. The exponent is normalized and bounded such that $0<\lambda_{k} A_{i j} \leq 1$.

As described above, MART has its disadvantage when it is applied to reconstruct the electron density distribution in the probed region. To resolve the problem, we introduce additional equations and include them as constraints in this research. These constraints impose smoothness on the IED field. The additional equations can be expressed in a matrix notation as:

$$
B_{l \times n} x_{n \times 1}=0 .
$$




\begin{tabular}{|c|c|c|}
\hline 1 & 2 & 3 \\
\hline 4 & 5 & 6 \\
\hline 7 & 8 & 9 \\
\hline
\end{tabular}

Figure 1. The sketch for creating of two-dimensional constraint matrix.

The selection of the matrix B is very important in equation (5). Generally, any physically reasonable information, expressed in the form of a finite difference equation, can be transferred. Treating the ionosphere as a smooth field is a reasonable way to simplify the discussion. A popular two-dimensional nine-point finite difference approximation of the second order Laplacian operator is used throughout in this work.

Figure 1 shows the representative sketch for creating a two-dimensional constraint matrix. According to the basic constraint rules, for those pixels that lie at the center of the probed region (i.e., 5th pixel in figure 1), the constraint is given by using the following two-dimensional nine-point finite difference approximation of the second order Laplacian operator:

$$
L_{0}=\begin{array}{|r|r|r|}
\hline-1 & -1 & -1 \\
\hline-1 & 8 & -1 \\
\hline-1 & -1 & -1 \\
\hline
\end{array}
$$

The operator has to be adjusted accordingly when the pixels locate at the edge of the probed region. For those pixels lying on the four corners of each layer, the operators are given in $L_{1}$ (i.e., the 7 th pixel in figure 1), $L_{2}$ (i.e., the 9 th pixel in figure 1), $L_{3}$ (i.e., the first pixel in figure 1 ) and $L_{4}$ (i.e., the third pixel in figure 1), respectively.

$$
\begin{aligned}
& L_{1}=\begin{array}{|r|r|}
\hline-1 & -1 \\
\hline 3 & -1 \\
\hline
\end{array} \\
& L_{2}=\begin{array}{|r|r|}
\hline-1 & -1 \\
\hline-1 & 3 \\
\hline
\end{array} \\
& L_{3}=\begin{array}{|r|r|}
\hline 3 & -1 \\
\hline-1 & -1 \\
\hline
\end{array} \\
& L_{4}=\begin{array}{|r|r|}
\hline-1 & 3 \\
\hline-1 & -1 \\
\hline
\end{array}
\end{aligned}
$$

However, for those pixels lying on the edge rather than the corner, the operators are given in $L_{5}$ (i.e., the 8 th pixel in figure 1 ), $L_{6}$ (i.e., the 2 nd pixel in figure 1), $L_{7}$ (i.e., the 4 th pixel in figure 1) and $L_{8}$ (i.e., the 6 th pixel in figure 1 ), respectively.

$$
\begin{aligned}
& L_{5}=\begin{array}{|r|r|r|}
\hline-1 & -1 & -1 \\
\hline-1 & 5 & -1 \\
\hline
\end{array} \\
& L_{6}=\begin{array}{|r|r|r|}
\hline-1 & 5 & -1 \\
\hline-1 & -1 & -1 \\
\hline
\end{array} \\
& L_{7}=\begin{array}{|r|r|}
\hline-1 & -1 \\
\hline 5 & -1 \\
\hline-1 & -1 \\
\hline
\end{array} \\
& L_{8}=\begin{array}{|r|r|}
\hline-1 & -1 \\
\hline-1 & 5 \\
\hline-1 & -1 \\
\hline
\end{array}
\end{aligned}
$$

As for other layers, the operators are given by using the above rules. According to the operators, the constrained matrix $\mathrm{B}$ in equation (5) can be created reasonably.

\section{Numerical simulation experiment}

To examine the feasibility and superiority of the new method in comparison with the MART algorithm, a simulation scheme is devised. The simulation procedure was as follows:

- In this simulated experiment, the latitude ranged from $30^{\circ} \mathrm{N}$ to $40^{\circ} \mathrm{N}$, the longitude ranged from $115^{\circ} \mathrm{E}$ to $125^{\circ} \mathrm{E}$, and the height ranged from 100 to $1000 \mathrm{~km}$ in steps of $15 \mathrm{~km}$. The discretized interval in latitude and longitude is $0.5^{\circ}$ and $1^{\circ}$, respectively.

- In a selected reference frame and time period, the actual positions of GNSS satellites and available 52 ground observation stations are used to create the projection matrix A.

- The IED at the center of each pixel is generated from the IRI2007 model and considered as the mean IED value of the corresponding pixel. The simulated time period is 05:40-06:00UT, 10 April 2009. The discrete density distribution is represented by $x_{\text {simu }}$, and then the simulated TEC value $y_{\text {simu }}$ without noise is computed by using the following equation:

$$
y_{\text {simu }}=A \cdot x_{\text {simu }} .
$$

- Taking the reality into account, a small amount of random noise $e_{\text {simu }}$ should be added to the simulated TEC values $y_{\text {simu }}$. One can then obtain:

$$
y_{\text {nois }}=y_{\text {simu }}+e_{\text {simu }}
$$

- CMART, MART and CA are used to invert the IED from the simulated TEC with noise in equation (7), respectively. The final estimate $x_{\mathrm{est}}$ is then obtained. 

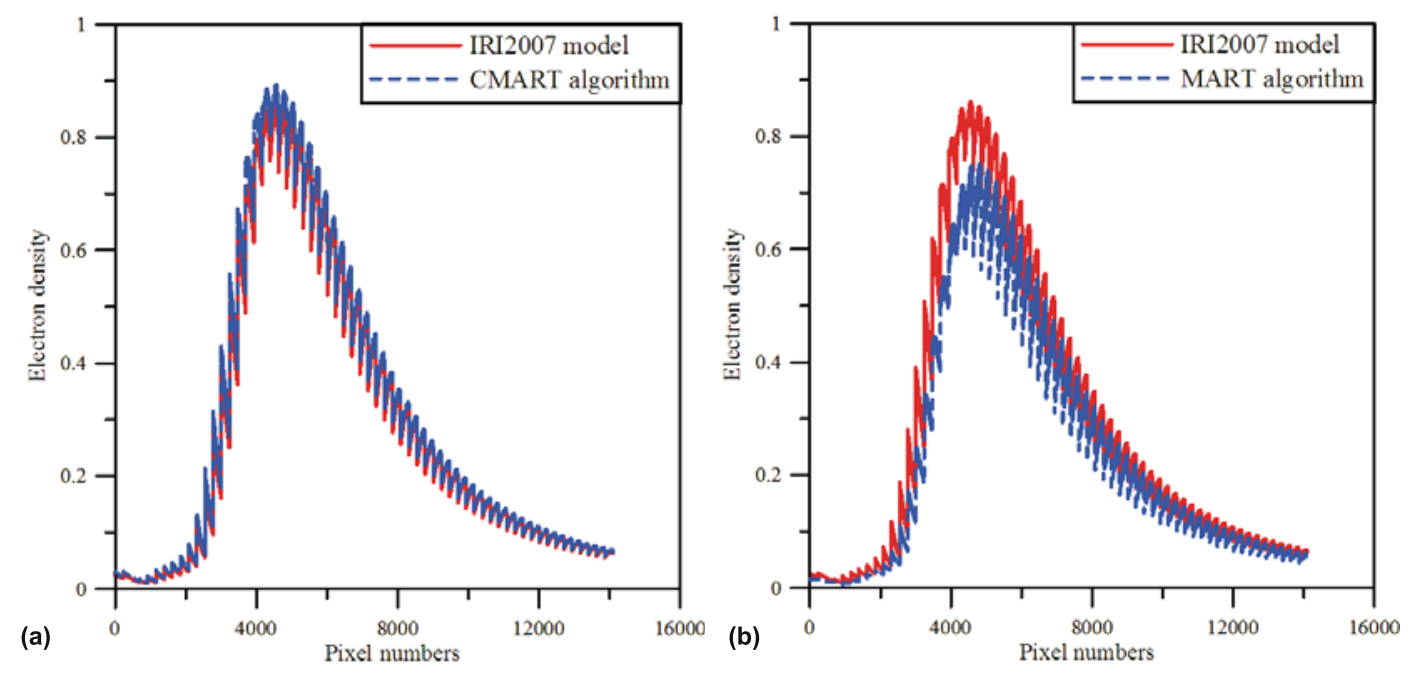

Figure 2. Comparison between the electron density distribution of the model data (blue) and the corresponding distributions of the electron density reconstructed by the two algorithms (red) (a) CMART and (b) MART. The unit of ionospheric electron density is $10^{11} \mathrm{e} / \mathrm{m}^{3}$.

According to the above interval, the probed region is discretized into 14091 pixels. Figure 2(a and b) shows the comparisons between the tomographic results of CMART and MART with those obtained from IRI2007 model, respectively. From figure 2(a), it can be seen that the tomogrpahic results of CMART algorithm agree better than those obtained from MART algorithm, the retrieval of electron density distribution in the reconstructed region was very similar to that given by IRI2007 model, and the density values were modified with the information from the neighbouring ones by the Laplacian constraint as expected. This demonstrated the feasibility of CMART to reconstruct IED distribution. The average density error of CMART reconstruction is $7.4 \times 10^{9} \mathrm{e} / \mathrm{m}^{3}$, which is very small compared with the peak density of $8.97 \times 10^{11} \mathrm{e} / \mathrm{m}^{3}$. However, the average density of MART is $7.72 \times 10^{10} \mathrm{e} / \mathrm{m}^{3}$, which is larger than the average error of CMART. The error comparison of two reconstruction algorithms validated that CMART was superior to MART algorithm.

To simplify the description, in the following section, one-iteration means that all available rays are used to update the reconstruction once. Figure 3 shows the comparisons between the images obtained from IRI 2007 model and the reconstructed results of five-iteration of the CMART and the CA before the iteration converges. From figure 3, it can be seen that the reconstructed image of the CMART is closer to the image obtained from IRI 2007 model than that reconstructed from the CA. The comparison validates that the CMART has the higher computational efficiency and the better reconstruction quality than the CA.
Figure 4 illustrates the comparisons between the images obtained from IRI 2007 model and the reconstructed results of the CMART and the CA. Figure 4(b) represents the reconstructed image of ten-iteration of the CMART, and figure 4(c) shows the reconstructed results of twenty-three iteration of the CA. From figure 4, we can see that the reconstructed image of ten-iteration of the CMART is similar to the image obtained from IRI 2007 model. However, the CA consumes twenty-three iterations. This fact further demonstrates that the CMART is higher than the method adopted by Pryse et al (1998) in computational efficiency.

\section{IED reconstruction based on actual GNSS observations}

\subsection{Tomographic reconstruction of IED during geomagnetic quiet day}

In this section, CMART was applied to the actual GNSS observation data obtained from 60 different receivers of Continuously Operating Reference System (CORS) of Jiangsu province and Shanghai city. Observation data obtained within 30 minutes every hour on 22 December 2008 are analyzed to investigate the effects of diurnal variations in the ionosphere. Some examples of the diurnal variations of the IED distribution at $116.5^{\circ} \mathrm{E}$ are shown in figure 5. The altitude ranges from 100 to $1000 \mathrm{~km}$ in the actual computation. Taking the small variation of electron density value between 700 and $1000 \mathrm{~km}$ into account, the altitude ranges from 100 to $700 \mathrm{~km}$ in the figure 5 . From figure 5, we can see that the IED reaches the maximum at 

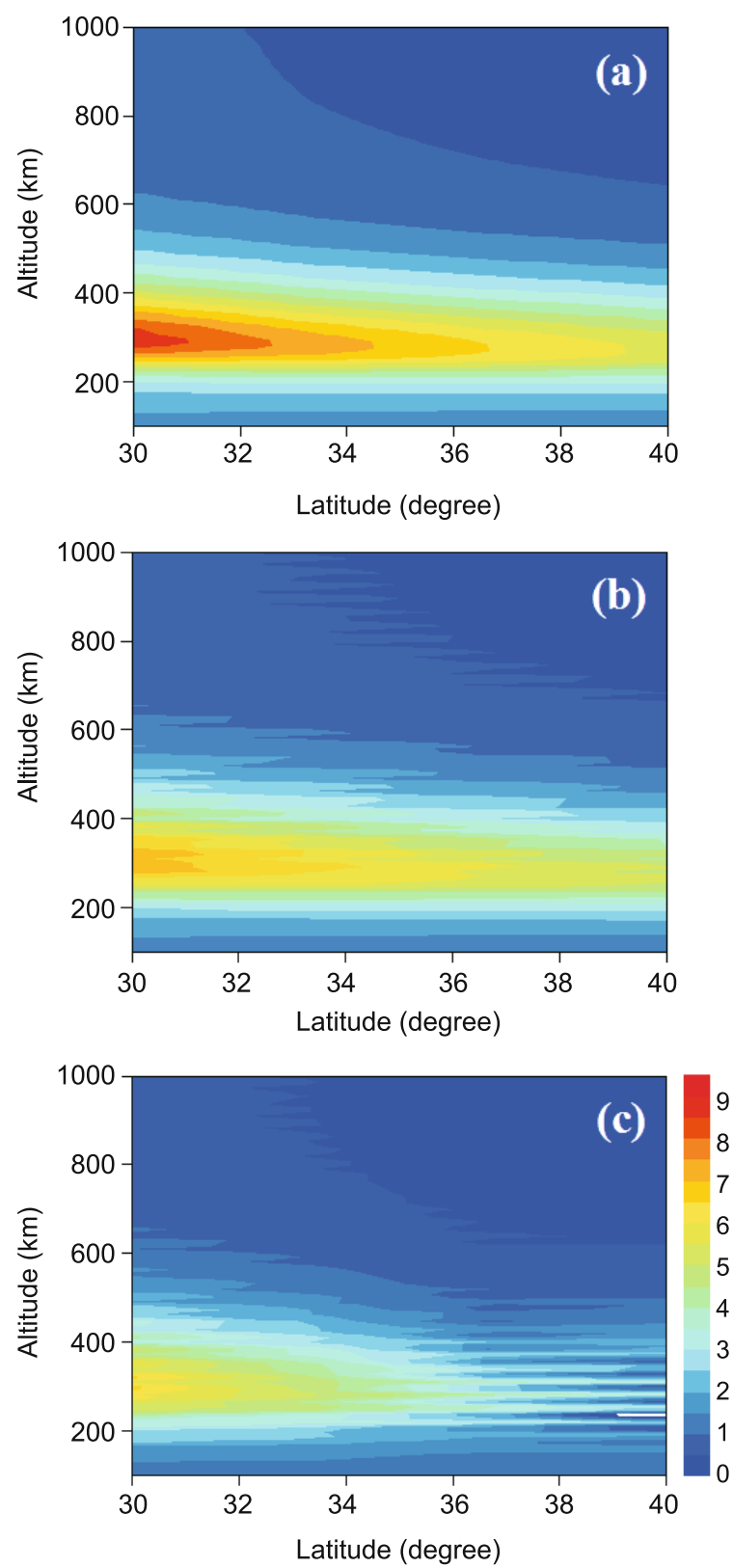

Figure 3. Numerical ionospheric model and images reconstructed through five-iteration by the CMART and the CA. The unit of IED is $10^{11} \mathrm{e} / \mathrm{m}^{3}$. (a) Original IED image from the IRI 2007 model; (b) reconstructed image using CMART algorithm; and (c) reconstructed image using CA.

05:00 UT (13:00 local time (LT)) and the minimum occurs at 21:00 UT (05:00 LT). The IED distributions in the south are larger than those in the north from 01:00 UT (09:00 LT) to 09:00 UT (17:00 LT). At 13:00 UT (21:00 LT), the IED values in the south tends to coincide with those in the north, and then the IED distributions in the south are smaller than those in the north from 17:00 UT (01:00 LT) to 21:00 UT (05:00 LT). It reflects the fact that there exists a close relationship between the variations of IED and the latitude. Meanwhile, it can also be seen that the distribution of IED
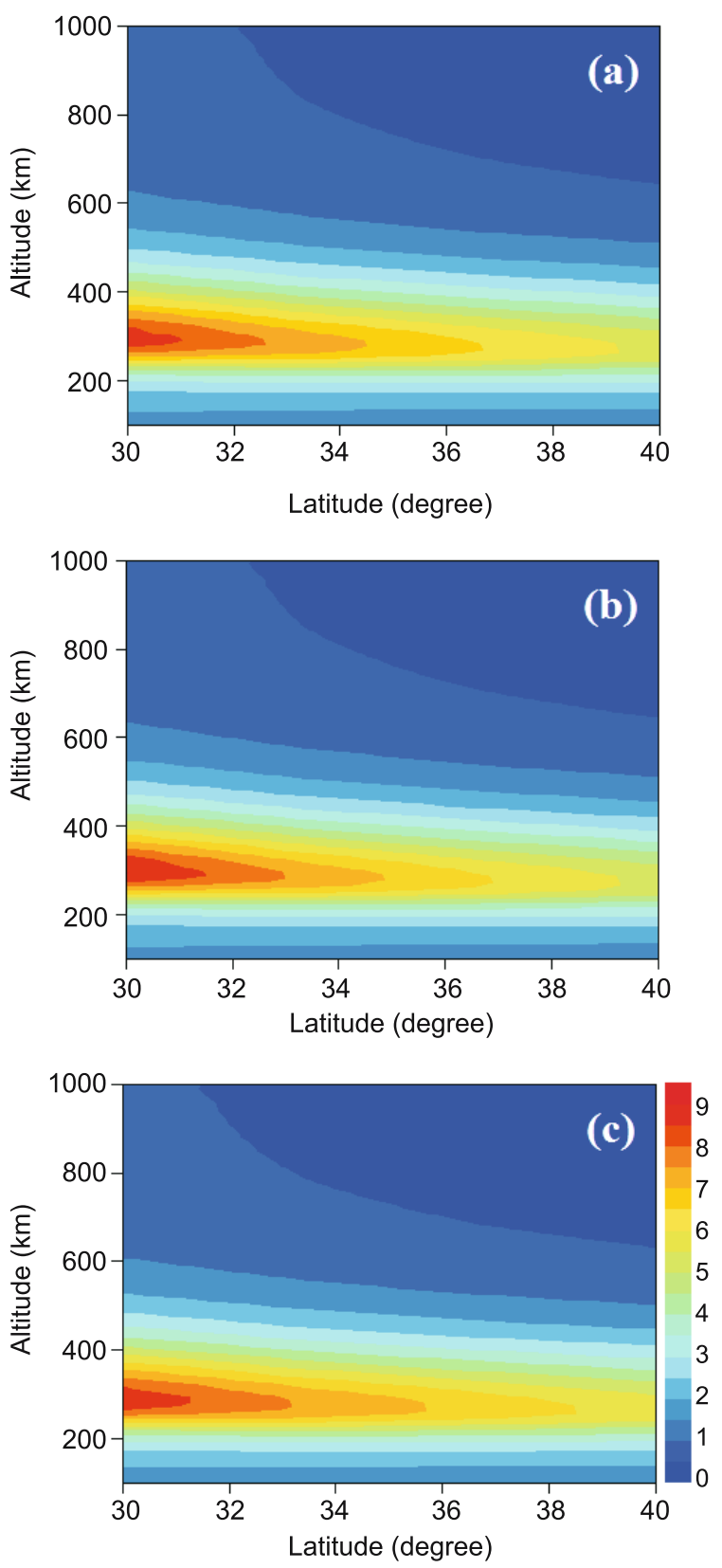

Figure 4. Numerical ionospheric model and images reconstructed after iteration is converged by using the CMART and the CA. The unit of IED is $10^{11} \mathrm{e} / \mathrm{m}^{3}$. (a) Original IED image from the IRI 2007 model; (b) reconstructed image of ten iterations using CMART; and (c) reconstructed image of twenty-three iterations using CA.

varies from small to large value, and then it varies from large to small value. Using this new method proposed by this paper, the IED distributions of other days in 2008 are reconstructed, and the above rules can also be verified.

Figure 6 shows a comparison of GNSS-derived and ionosonde-derived vertical electron density profiles over Beijing ionosonde station in China at 05:00 UT and 09:00 UT. The comparisons demonstrated the reliability of the tomographic results based on CMART by using actual GNSS 

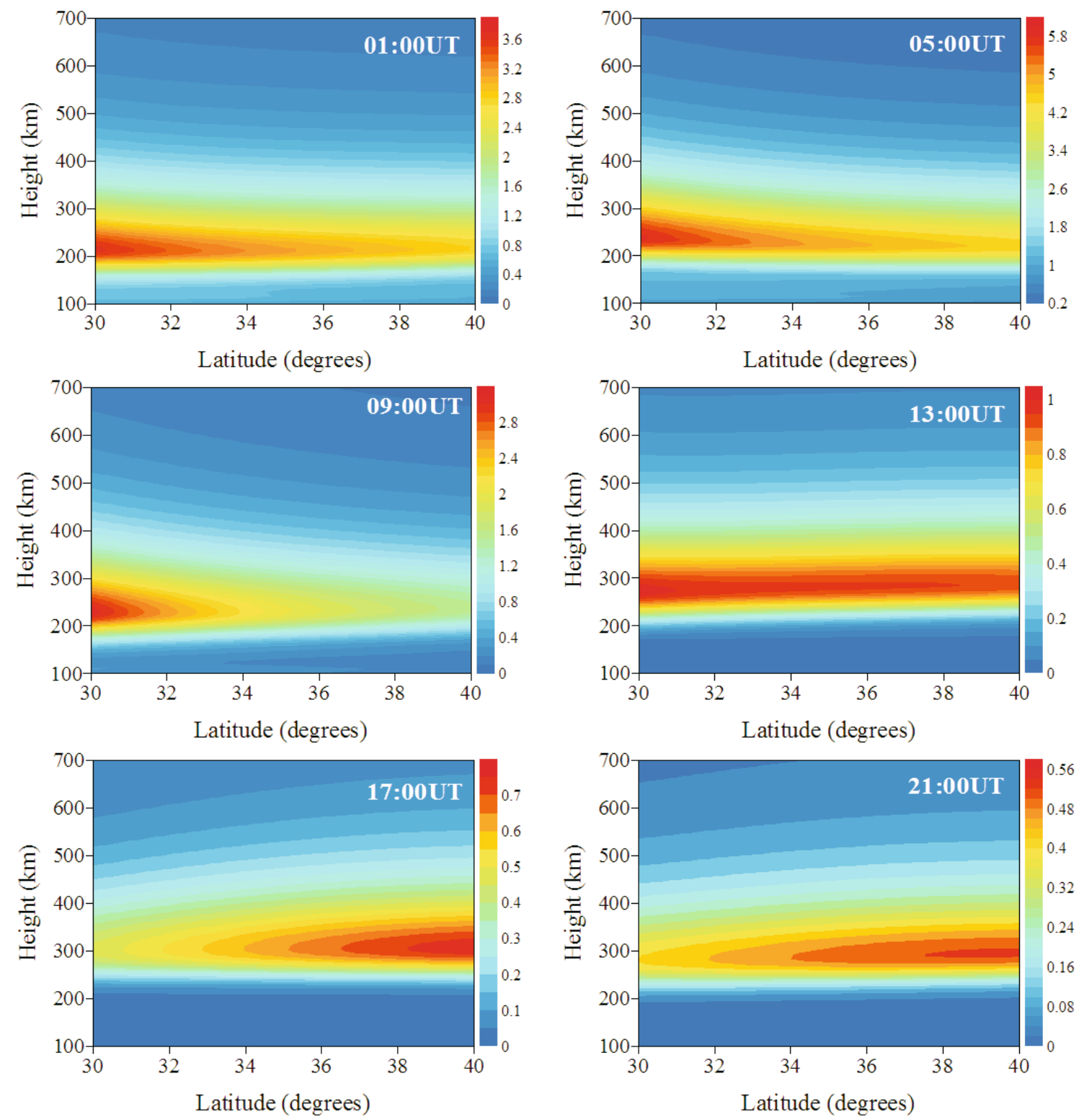

Figure 5. Sample of IED distribution obtained from 22 November 2008. Each subfigure represents the corresponding electron density reconstructed in different local time which is labeled at the subfigure. The unit of IED is $10^{11} \mathrm{e} / \mathrm{m}^{3}$.

observations. Meanwhile, it can be seen that the profiles obtained from CMART are more close to the ionosonde than those from MART as a whole. This fact further validated the superiority of CMART to the MART.

\section{Tomographic reconstruction of IED during geomagnetic storm}

In order to further demonstrate the performance of CMART under geomagnetic disturbance condition, the GPS data on October 20, 2003 were used to reconstruct the IED distribution. Interest is focused on this day since a severe geomagnetic storm event happened. The maximum of $K p$ index is 9 and the minimum is -5 . Figure 7 showed the reconstruction images of IED distribution for three time periods on this day. From the images, it can be seen that the obvious disturbed structure of the ionosphere appeared during geomagnetic storm occurring. This demonstrated that the CMART can also be used to handle perturbation.

\section{Conclusions}

A constrained MART algorithm is introduced for IED tomographic reconstruction. The CMART imposes a constraint according to the smoothness of neighbour pixels and overcomes the disadvantages of the MART to some extent. Numerical 

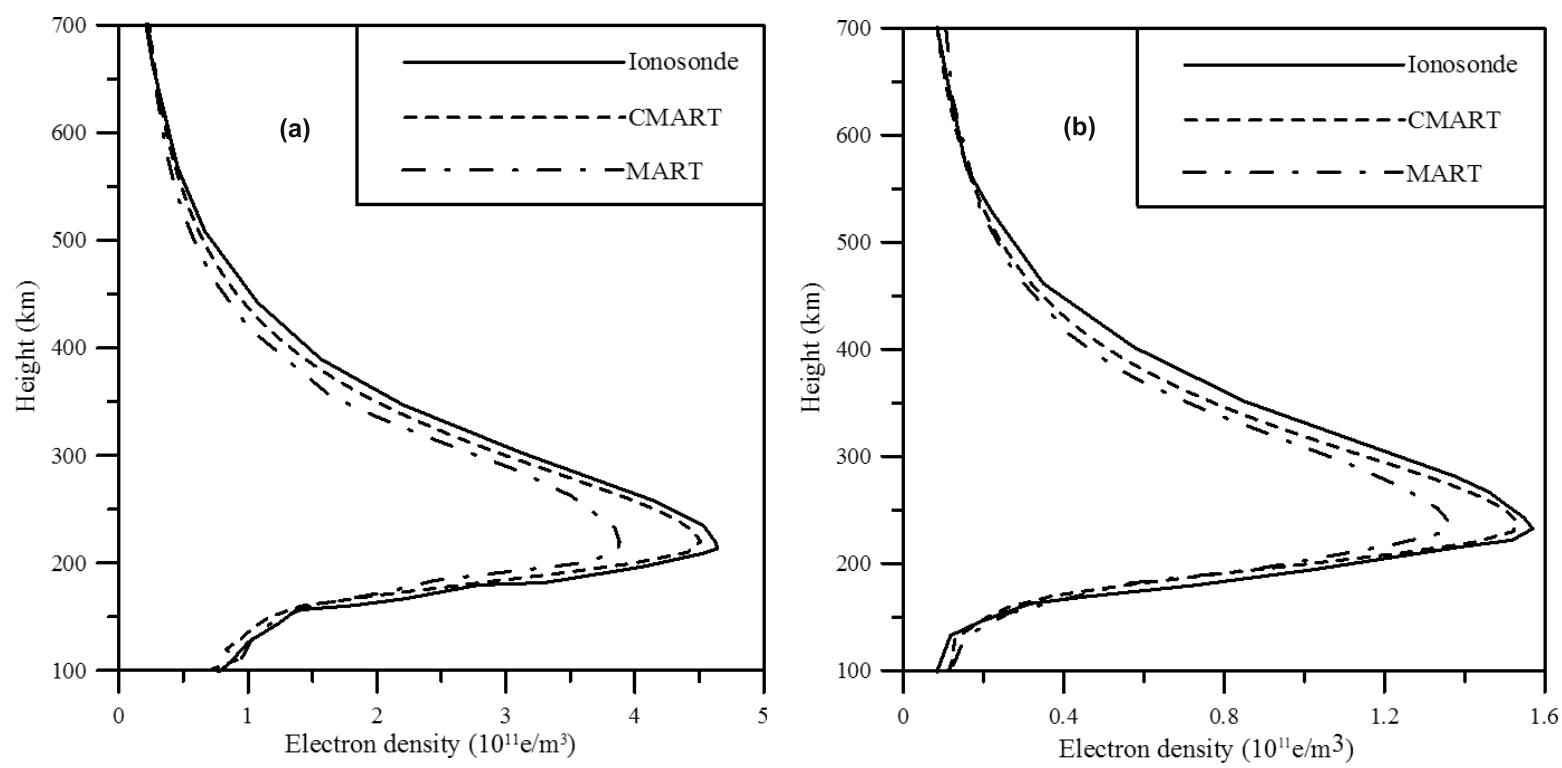

Figure 6. Comparison among the vertical electron density distribution of the ionosonde and the corresponding distribution of the electron density reconstructed by CMART and MART. (a) 05:00 UT; and (b) 09:00 UT. The unit of IED is $10^{11} \mathrm{e} / \mathrm{m}^{3}$.
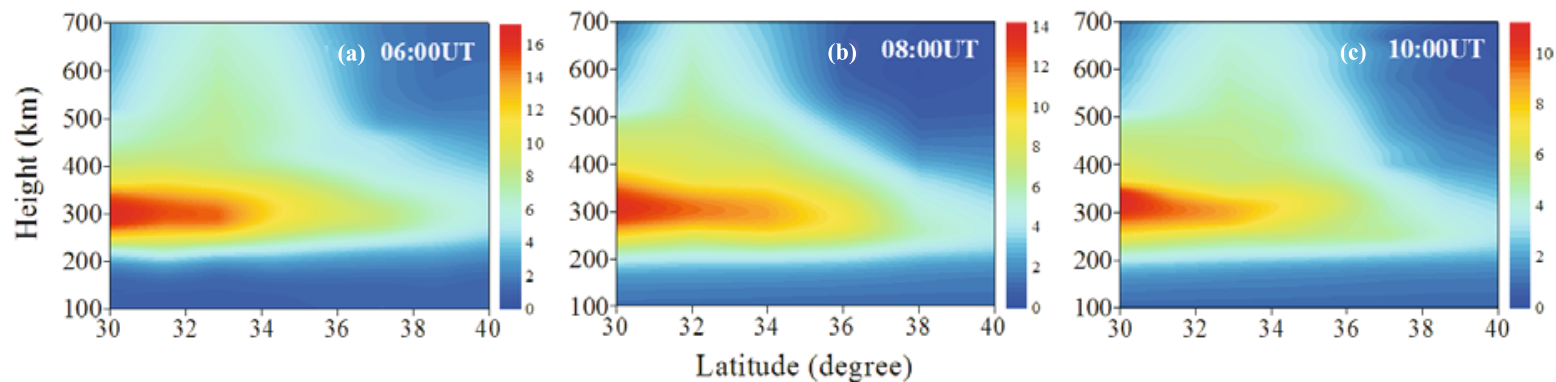

Figure 7. Variations of IED at longitude of $116^{\circ} \mathrm{E}$ for the three time periods on October 30, 2003. (a) 05:30-06:00 UT (13:30-14:00 LT), (b) 07:30-08:00 UT (15:30-16:00 LT) and (c) 09:30-10:00 UT (17:30-18:00 LT). The unit of IED is $10^{11} \mathrm{e} / \mathrm{m}^{3}$.

simulations based IRI2007 model were presented and the feasibility of the new method is validated, and then we showed that this new method is superior to the conventional MART algorithm using numerical simulation. The accuracy of IED obtained from CMART is obviously improved, and the computational efficiency of the CMART is higher than the CA. Finally, the new method is successfully applied to the tomographic reconstruction of IED distributions using actual GNSS observation data from the CORS of jiangsu province and Shanghai city. Meanwhile, the CMART is demonstrated to be applied to handle perturbation during geomagnetic storm occurring.

In this research, the effects of plasmasphere can not be considered. To evaluate the effects of plasmasphere on the reconstructed results, we need to further improve the model of the plasmasphere. In the future, the new method can be extended to four-dimensional tomographic reconstruction by considering the time evolution of the ionosphere.

\section{Acknowledgements}

This research is supported by the National Natural Science Foundation of China (Grant no. 40804002), the National Science Fund for Distinguished Young Scholars of China (Grant no. 40625013) and the Scientific Research Fund of Hunan Provincial Education Department (09B007). The authors would also like to thank the anonymous reviewers for their constructive suggestions towards the improvement of this paper.

\section{References}

Austen J R, Franke S J and Liu C H 1988 Ionospheric imaging using computerized tomography; Radio Sci. $\mathbf{2 3}$ 299-307.

Andreeva E S, Franke S J and Yeh K C 1990 Radio tomographic reconstruction of ionization dip in the plasma near the Earth; J. Exp. Theor. Phys. Lett. 52 145-148.

Garcia R and Crespon F 2008 Radio tomography of the ionophere: Analysis of an underdetermined, ill-posed 
inverse problem and regional application; Radio Sci. 43 RS2014, doi:10.1029/2007 RS003714.

Huang C S, Sofko G and Kelley M 1998 Numerical simulations of midlatitude ionospheric perturbations produced by gravity waves; J. Geophys. Res. 103 6977-6989.

Kak A and Slaney M 1988 Principles of computerized tomographic imaging; Soc. for Indust. and Appl. Math. Philadelphia, Pa.

Kunitake M, Ohtaka K, Maruyama T, Tokumaru M, Marioka A and Wantabe S 1995 Tomographic imaging of the ionosphere over Japan by the modified truncated SVD method; Ann. Geophys. 13 1303-1310.

Kunitsyn V E and Tereshchenko E D 1992 Radio tomography of the ionosphere; IEEE Antennas Propagat. Mag. $3422-32$.

Markkanen M, Lehtinen M, Nygren T, Pirttila J, Henelius P, Vilenius E, Tereshchenko E D and Lhuduk B Z 1995 Bayesian approach to satellite radio tomography with applications in the Scandinavian sector; Ann. Geophys. 13 1277-1287.

Mitchell C N, Jones D G, Kersley L, Pryse S E and Walker L K 1995 Imaging of field-aligned structures in the auroral ionosphere; Ann. Geophys. 13 1311-1319.

Pryse S E, Mitchell C N, Heaton J A T and Kersley L 1995 Traveling ionospheric disturbances imaged by tomographic techniques; Ann. Geophys. 13 1325-1342.
Pryse S E, Kersley S, Mitchell C N, Spencer P S J and Willams M J 1998 A comparison of reconstruction techniques used in ionospheric tomography; Radio Sci. 33 $1767-1779$.

Wen D B 2007 Imaging the ionospheric electron density using a combined tomographic algorithm; In: Proc. ION GNSS, FortWorth, TX, September, pp. 2337-2345.

Wen D B 2010 The research progress of computerized ionospheric tomography technique; Bull. Nat. Sci. Found. China 24 17-20.

Wen D B, Yuan Y B, Ou J K, Huo X L and Zhang K F 2007a Three dimensional ionospheric tomography by an improved algebraic reconstruction technique; GPS Solu. 11 251-258.

Wen D B, Yuan Y B and Ou J K 2007b Monitoring the three-dimensional ionospheric electron density distribution using GPS observations over China; J. Earth Syst. Sci. 116(3) 235-244.

Wen D B, Yuan Y B, Ou J K and Zhang K F 2008 A hybrid reconstruction algorithm for three dimensional ionospheric tomography; IEEE Trans. Geosci. Remote Sensing 46 1733-1738.

Zhai Y and Cumme S 2005 A flexible and robust direct reconstruction method for agnetospheric radio tomography; Radio Sci. 40 RS3004, doi: 10.1029/2004RS 003100 . 